\title{
Variation of patient imaging doses with scanning parameters for linac-integrated kilovoltage cone beam CT
}

\author{
Xiongfei Liao ${ }^{\mathrm{a}}$, Yunlai Wang, ${ }^{\mathrm{b},}$, Jinyi Lang ${ }^{\mathrm{a}}$, Pei Wang ${ }^{\mathrm{a}}, \mathrm{Jie} \mathrm{Li}^{\mathrm{a}}$, Ruigang Ge $\mathrm{b}^{\mathrm{b}}$ and Jack Yang ${ }^{\mathrm{c}}$ \\ ${ }^{a}$ Department of Radiation Oncology, Sichuan Cancer Hospital, 55 4th Section Renmin South Road, \\ Chengdu, 610041, China \\ ${ }^{\mathrm{b}}$ Department of Radiation Oncology, Chinese PLA General Hospital, 28 Fuxing Avenue, Beijing, \\ 100853, China \\ ${ }^{\mathrm{c}}$ Department of Radiation Oncology, Monmouth Medical Center, Long Branch, NJ 07740, USA
}

\begin{abstract}
To evaluate the Elekta kilovoltage CBCT doses and the associated technical protocols with patient dosimetry estimation. Image guidance technique with cone-beam CT (CBCT) in radiation oncology on a daily basis can deliver a significant dose to the patient. To evaluate the patient dose from LINAC-integrated $\mathrm{kV}$ cone beam CT imaging in imageguided radiotherapy. CT dose index (CTDI) were measured with PTW TM30009 CT ion chamber in air, in head phantom and body phantom, respectively; with different combinations of tube voltage, current, exposure time per frame, collimator and gantry rotation range. Dose length products (DLP) were subsequently calculated to account for volume integration effects. The CTDI and DLP were also compared to $\mathrm{AcQSim}^{\mathrm{TM}}$ simulator CT for routine clinical protocols. Both CTDI $\mathrm{Cir}_{\text {and }}$ $\mathrm{CTDI}_{\mathrm{w}}$ depended quadratically on the voltage, while linearly on milliampere x seconds (mAs) settings. It was shown that $\mathrm{CTDI}_{\mathrm{w}}$ and DLP had very close relationship with the collimator settings and the gantry rotation ranges. Normalized CTDI for Elekta XVI ${ }^{\mathrm{TM}}$ CBCT was lower than that of ACQSim simulator CT owing to its pulsed radiation output characteristics. $\mathrm{CTDI}_{\mathrm{w}}$ can be used to assess the patient dose in CBCT due to its simplicity for measurement and reproducibility. Regular measurement should be performed in QA \& QC program. Optimal image parameters should be chosen to reduce patient dose during CBCT.
\end{abstract}

Keywords: Cone-beam CT, CT dose index, dose-length product, image-guided radiotherapy

\section{Introduction}

Intensity-modulated radiotherapy (IMRT) has the ability to create highly conformal dose distributions for target volume dose coverage and normal tissue avoidance. Usage of IMRT for radiation treatment has been widely adopted for more than one decade for better customized dosimetry results. The improved dose conformity and sharp dose gradient have necessitated accurate beam targeting technique for treatment delivery. However, there are many components affecting the reproducibility of target volume and location during and between subsequent treatment fractions,

\footnotetext{
* Address for correspondence: Yunlai Wang, Department of Radiation Oncology, Chinese PLA General Hospital, 28 Fuxing Avenue, Beijing, 100853, China. Tel.: +86 13552028796; Fax: +86 1066937762; E-mail: nanyangwang@163.com.
} 
including displacement of patient position and organ motions inter-fractionally and inter-fractionally [1-3].

Cone-beam computed tomography (CBCT) integrated with the LINAC is used for patient positioning during radiation therapy. Functionality of $\mathrm{CBCT}$ is to create the Image Guided Radiation Therapy (IGRT) process in order to reduce patient setup errors and/or assess change of tumor volume in time. By registering the planning $\mathrm{CT}$ along with the $\mathrm{CBCT}$ images, users can determine the appropriate shifts of the treatment table required to properly align the patient. However, the added benefit of improved patient positioning comes at the cost of additional radiation dose, which in a high fraction regimen with daily imaging can lead to a total dose on the order of a Gray (Gy) or more for a number of organs if those extra radiation from CBCT is counted for [4-9].

However, due to the $\mathrm{X}$-ray radiation nature of the $\mathrm{CBCT}$ process, dose deposited in patient during the daily procedures in patient repositioning has become a technical issue to recognize the extra dose received on top of treatment dosage [10-16]. In an attempt to determine what level of optimization of scanning parameters is taking place, the main purpose of the current study is to evaluate the clinical impact of these changes on the doses received by patients undergoing IGRT and to assess the associate image quality. While it is important to improve the treatment delivery accuracy, it is also necessary to discover the balance in between the dose and image quality with optimized scanning parameters.

The extra CT radiation exposure for IGRT possessed some potential radiation risks to patients. Therefore, it is quite necessary to evaluate the patient dose from CBCT scanning in order to optimize the imaging acquisition parameters and to reduce the patient dose simultaneously. The CBCT scanning characteristics differed from those in conventional CT, therefore, specific techniques were necessary to be explored in order to allow detailed assessment of patient dose in CBCT. In this study, we will be using the common CT dose Index (CTDI) for evaluation purpose since it is the wellestablished dose indicator defined to represent the integrated dose to one specific point in an axial scan $[17,18]$.

\section{Materials and methods}

\subsection{X-ray volume imaging (XVI) CBCT system}

The XVI 3.5 CBCT unit is quipped and integrated on Elekta Synergy accelerator. It consists of retractable X-ray generator and amorphous silicon panel detector, and is mounted orthogonally to the MV beam line of the accelerator. Volumetric images are reconstructed simultaneously with the rotational acquisition. The CBCT images are manually or automatically registered with the planning CT image set, differences between reference and local geometry are calculated and displayed as translation along and rotation about the three main axes. XVI has three modes of collimators labeled $\mathrm{S}$ (small), M (medium) and L (large), which is different in width and length dimensions, referring to the field of view (FOV). The X-ray generator has a focal spot size of $1.2 \mathrm{~mm}^{2}$, located $100 \mathrm{~cm}$ from isocenter of the LINAC. The tube has $1.5 \mathrm{~mm}$ Al equivalent inherent filtration and additional compound filtration with $1.5 \mathrm{~mm} \mathrm{Al}$ and $0.127 \mathrm{~mm} \mathrm{Cu}$, totally is equivalent to $3.6 \mathrm{~mm} \mathrm{Al}$. Tube potentials range from $60 \mathrm{kV}$ to $160 \mathrm{kV}$. Pulsed radiation exposures range from $0.1 \mathrm{mAs}$ to $3.2 \mathrm{mAs}$ per projection. The detector is a $\mathrm{Gd} 2 \mathrm{O} 2 \mathrm{~S}$ : $\mathrm{Tb}$ amorphous silicon $(\mathrm{aSi})$ flat panel with an active area of $41 \mathrm{~cm} \times 41 \mathrm{~cm}$ addressed as an array of $1024 \times 1024$ pixels. The panel is located at $153.6 \mathrm{~cm}$ from the source. 
A typical imaging sequence with approximately $650 \mathrm{CBCT}$ projections was acquired during a $360^{\circ}$ gantry rotation and was utilized for three-dimensional reconstruction of the irradiated volume. The Xray source characteristics for a single projection were $120 \mathrm{kV}, 25 \mathrm{~mA}$ and $40 \mathrm{~ms}$, which results in approximately a total of $650 \mathrm{mAs}$ in a full scan.

\subsection{Free-in-air measurements}

The free-in-air CTDI was determined from measurements by exposing a CT pencil ionization chamber (PTW TM30009) without any build up materials around. This chamber consisted of total $3.14 \mathrm{cc}$ sensitive volume and a measuring length of $10 \mathrm{~cm}$. As the irradiated volume is longer than 10 $\mathrm{cm}$, both chambers were both irradiated over the full length. The dose measured with the CT-chamber was divided by 10 to calculate the effective CTDI for $1 \mathrm{~cm}$ length. Air density corrections were made by typing in air pressure and the temperature of treatment room. $\mathrm{CTDI}_{\text {air }}$ was then calculated using:

$$
C T D I_{\text {air }}=R \cdot N_{x} / t
$$

where $\mathrm{R}(\mathrm{mGy}-\mathrm{cm})$ is the electrometer reading corrected for room temperature and air pressure, $\mathrm{N}_{\mathrm{x}}$ is the chamber calibration factor (absorbed dose in air), $\mathrm{t}$ is the pencil chamber length in $\mathrm{cm}$ if the collimator length is greater than the pencil chamber active length, otherwise, it is the nominal collimator length itself.

For comparisons, the air kerma measured with $0.6 \mathrm{~cm}^{3}$ ionization chamber (PTW 31013) can be expressed as:

$$
K_{\text {air }}=R \cdot N_{x}
$$

Where the length factor has been eliminated since the collecting volume is small

\subsection{Measurements in phantom}

For the CTDI measurements, acrylic cylindrical phantoms with a diameter of $16 \mathrm{~cm}$ (PTW T40017) and $32 \mathrm{~cm}$ (PTW T40016), both with $15 \mathrm{~cm}$ height were utilized. Both phantoms have five holes to place an ionization chamber, one in the center and the others at $0^{\circ}, 90^{\circ}, 180^{\circ}$ and $270^{\circ}$ each with a distance of $1 \mathrm{~cm}$ away to the surface of the phantom in 4 quadrants. Dose measurements were accomplished in all five positions with a volume scan.

The phantom was positioned on the table top in the bracket which keeps the phantom in place, and exactly aligned the etched crosshairs on the phantom using the room lasers. The CT pencil ionization chamber was then inserted into one of five holes of the head or body phantom. During measurements, the other holes were filled by acrylic dummy plugs when not used. Three measurements were carried out to give an acceptable mean value. The doses in the center of the phantoms $\left(\mathrm{CTDI}_{\mathrm{c}}\right)$ and in four peripheral points were measured. The peripheral value $\left(\mathrm{CTDI}_{\mathrm{p}}\right)$ is calculated as the mean dose at four points orthogonal on the phantom. The weighted $\mathrm{CTDI}_{\mathrm{w}}$ is then calculated from the central and peripheral dose measurements as follows [17]:

$$
C T D I_{w}=\frac{1}{3} C T D I_{c}+\frac{2}{3} C T D I_{p}
$$


The dose length product (DLP) represents the exposure for a complete scan. It is calculated from the following equation:

$$
D L P=C T D I_{w} \cdot L
$$

Where $L$ is the scanning length, namely the length of the collimator.

Different combinations of preset parameters, such as voltage, current, collimator, and gantry rotation range, were used to evaluate the effect of imaging parameters on the patient dose. The total mAs is calculated as following:

$\mathrm{mAs}$ is defined as the (nominal projection number $) \times($ current $(\mathrm{mA})) \times($ exposure time $(\mathrm{ms}))$ per projection.

For routine scanning protocols, $\mathrm{CTDI}_{\mathrm{w}}$ and $\mathrm{DLP}$ of $\mathrm{kV}$ XVI cone beam CT were compared to corresponding results from Philips Brilliance simulator CT.

\section{Results}

\subsection{CTDIair and kair}

The preset for free-in-air CTDI measurements was $120 \mathrm{kV}, 10 \mathrm{~mA}$ and $10 \mathrm{~ms}$ per frame. The variation of CTDIair with collimators was shown in Table 1.

It was discovered that when the scan length was larger than the active length of CT pencil chamber, the $\mathrm{CTDI}_{\text {air }}$ value was very close to $\mathrm{K}_{\text {air }}$, meaning both $\mathrm{CT}$ pencil chamber and $0.6 \mathrm{cc}$ Farmer type chamber have measured the equilibrium dose, in the collecting volume . But if the scanning length was larger than the length of CT pencil chamber, then the $\mathrm{CTDI}_{\text {air }}$ was larger than the value of $\mathrm{K}_{\text {air }}$. This is due to the process that CT pencil chamber has integrated more scattering radiation into the collecting volume from the specific chamber geometry. The radiation output changes minimally with the size of the collimator according to the testing results shown in Table 1.

\section{2. $C T D I_{w}$}

With scanning preset of S20 collimator, the $10 \mathrm{~mA}, 10 \mathrm{~ms}$ exposure time per frame, with voltage changed from $80 \mathrm{kV}$ to $140 \mathrm{kV}$, the measured $\mathrm{CTDI}_{\mathrm{w}}$ in head and body phantoms both depended quadratically on the voltage, as showed in Figure 1. Fitted relationship between $\mathrm{CTDI}_{\mathrm{w}}$ and voltage were calculated as follows:

Table 1

\begin{tabular}{lll} 
Variation of $\mathrm{CTDI}_{\text {air }}$ with the collimator delivered at & \multicolumn{2}{c}{$\mathrm{mA}, 10 \mathrm{~ms}$ at $120 \mathrm{kV}$} \\
\hline collimator & $\mathrm{CTDI}_{\text {air }}(\mathrm{mGy})$ & $\mathrm{K}_{\text {air }}(\mathrm{mGy})$ \\
\hline S20 & 2.04 & 2.01 \\
M20 & 2.03 & 2.01 \\
M10 & 1.98 & 1.93 \\
M2 & 2.0 & 1.82 \\
L20 & 1.97 & 1.86 \\
L10 & 1.95 & 1.81 \\
L2 & 1.94 & 1.75 \\
\hline
\end{tabular}




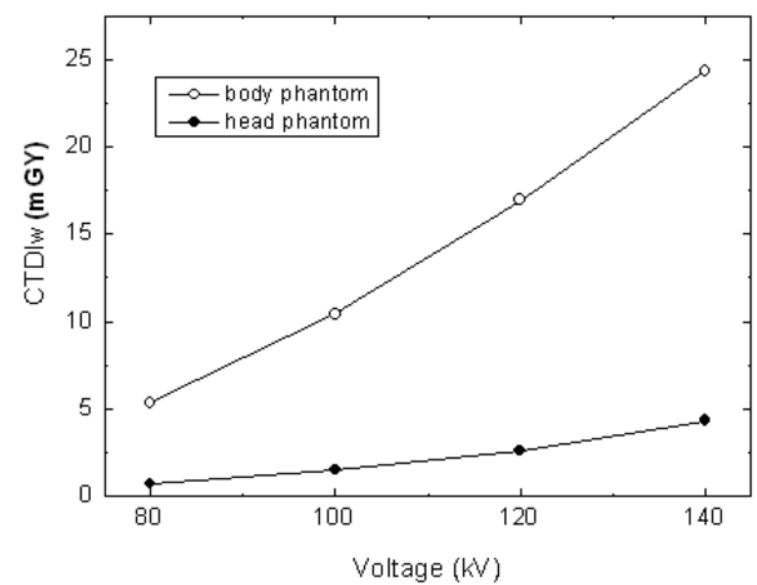

Fig. 1. $\mathrm{CTDI}_{\mathrm{w}}$ depends quadratically on the voltage both in head and body phantoms. Technical preset was: S20, $10 \mathrm{~mA}, 10$ ms.

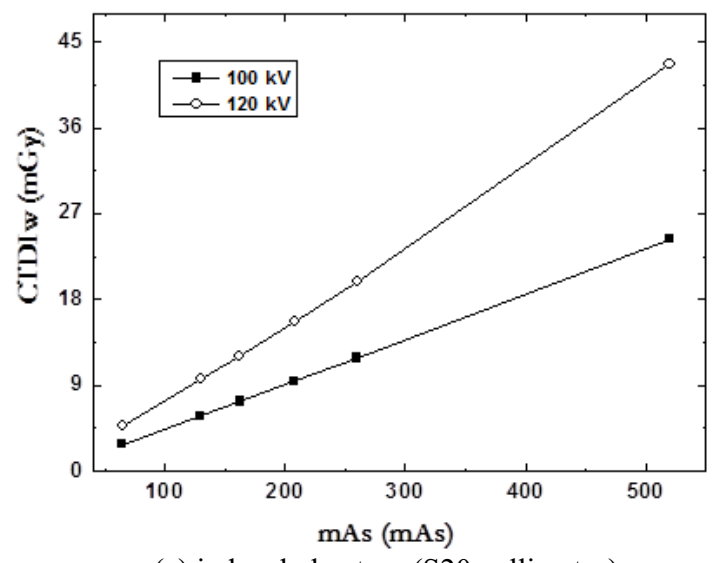

(a) in head phantom (S20 collimator)

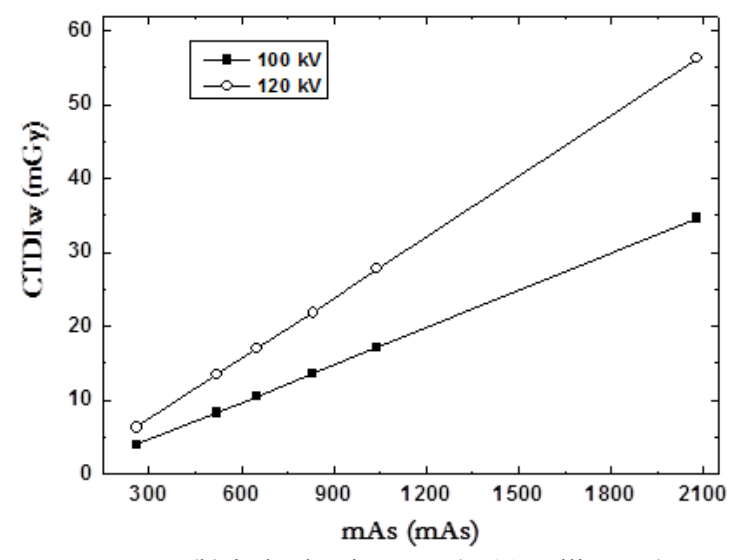

(b) in body phantom (M20 collimator)

Fig. 2. Linear variation of $\mathrm{CTDI}_{\mathrm{w}}$ with the product of current and exposure time both in head and body phantoms.

$$
\begin{aligned}
& C T D I_{w}=2.4955-0.0684 \cdot k V+0.0006 \cdot k V^{2} \text { (head phantom) } \\
& C T D I_{w}=-4.0365-0.0021 \cdot k V+0.0014 \cdot k V^{2} \text { (body phantom) }
\end{aligned}
$$

Linear relationship was found between $\mathrm{CTDI}_{\mathrm{w}}$ and $\mathrm{mAs}$, which followed the linear regression of $C T D I_{w}=-0.218+0.047 \cdot m A s$ in head phantom and $C T D I_{w}=-0.875+0.028 \cdot m A s$ in body phantom. The linear corelevant coefficients are greater than 0.99 , as showed in Figure 2.

The gantry and the X-ray generator rotate a complete circle (360 degrees) for a full scan. In comparison, the gantry rotates from 260 to 100 while the X-ray source from 350 to 190 is classified as half scan mode. For the preset of S20 collimator, $100 \mathrm{kV}, 10 \mathrm{~mA}$ and $10 \mathrm{~ms}$ per frame, the measured CTDI for full and half scan was shown in Figure 3.

The $\mathrm{CTDI}_{\mathrm{w}}$ value was numerically reduced from $1.50 \mathrm{mGy}$ for full scan to $0.86 \mathrm{mGy}$ for half scan due to the reduction of total frame number. In full scan mode, we observed that the lower peripheral CTDI was smaller than the upper one was owing to the additional attenuation effect of fiber couchtop, 


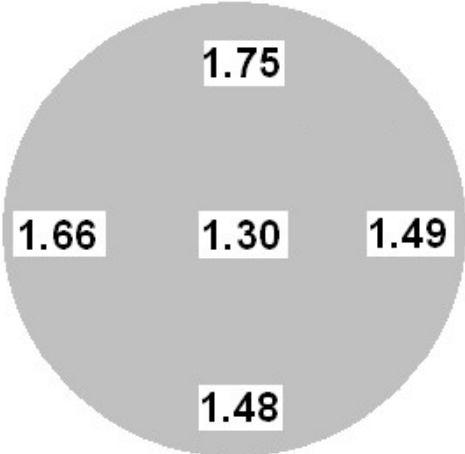

(a) Full scan

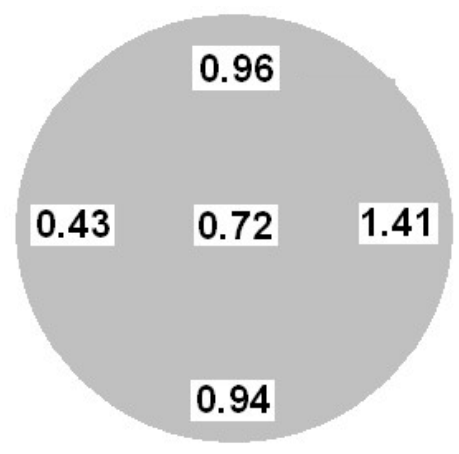

(b) Half scan

Fig. 3. Variation of $\mathrm{CTDI}_{\mathrm{w}}$ (in $\mathrm{mGy}$ ) in head phantom with the gantry rotation ranges.

while the left peripheral CTDI was larger than the right one was introduced by the rotation speed changes of gantry which was designed to turn slower at the end of the scan cycle. In contrast, the dose distribution was more inhomogeneous across the transversal plane due to the non-uniform irradiation pattern for CBCT. The ionization chamber was irradiated with different time intervals at four peripheral points during rotations. Therefore, the similar irradiation configuration has led to the result that the upper peripheral CTDI was close to the lower one.

The CTDI ${ }_{\mathrm{s}}$ were measured in body phantom with different collimators at a fixed scan preset of 40 $\mathrm{mA}$ and $25 \mathrm{~ms}$ per frame at $120 \mathrm{kV}$ with full scan mode. DLP were subsequently calculated according the length of collimator in longitudinal direction. The experimental results were shown in Table 2.

It was then discovered that the CTDI ${ }_{\mathrm{s}}$ and DLP changed considerably with the collimator settings. Although S20 and M20 have the same field size of $27.7 \mathrm{~cm} \times 27.7 \mathrm{~cm}, \mathrm{M} 20$ was designed to have a lateral field offset of $11.5 \mathrm{~cm}$ in order to acquire a reconstructed volume with $42.6 \mathrm{~cm}$ diameter; therefore, its $\mathrm{CTDI}_{\mathrm{p}}$ was lower than that of S20. M20, M10, and M2 have the similar scanning configuration, $\mathrm{CTDI}_{\mathrm{s}}$ and DLP were, however, quite different due to the output of field resulting from the length of the collimator.

\section{Discussion}

The weighted computed tomography dose index $\left(\mathrm{CTDI}_{\mathrm{w}}\right)$ is an approximation of average dose over a single slice in the standard head or body CT dosimetry phantom. Measurement of CTDI in the standard head or body CT dosimetry phantom can provide an indication of the average dose over a single slice for each setting of normal slice thickness.

CTDI and DLP depend largely on the preset scan parameters, such as X-ray tube voltage, current, exposure time per frame, collimator, et. Combination of optimized scanning parameters should be

Table 2

Variation of imaging dose with various collimator settings

\begin{tabular}{llllll}
\hline Collimator & Length $(\mathrm{cm})$ & CTDI $_{\mathrm{c}}(\mathrm{mGy})$ & CTDI $_{\mathrm{p}}(\mathrm{mGy})$ & CTDI $_{\mathrm{w}}(\mathrm{mGy})$ & DLP $(\mathrm{mGycm})$ \\
\hline S20 & 27.7 & 10.42 & 25.53 & 20.49 & 567.6 \\
M20 & 27.7 & 9.37 & 20.96 & 17.09 & 473.4 \\
M10 & 13.5 & 8.32 & 19.68 & 15.86 & 214.1 \\
M2 & 3.5 & 7.55 & 17.67 & 14.37 & 56.6 \\
\hline
\end{tabular}


properly chosen to reduce patient imaging dose while maintaining the same image quality [19-21]. In our another study [22] which shows that the spatial resolution increased from $51 \mathrm{p} / \mathrm{cm}$ to $71 \mathrm{p} / \mathrm{cm}$ when the tube voltage varied from $80 \mathrm{kV}$ to $140 \mathrm{kV}$. Current and exposure time per frame had little influence on the spatial resolution. The spatial resolution mainly depended on collimator, reconstruction resolution, and gantry rotation range. $\mathrm{CTDI}_{\mathrm{w}}$ has presented a quadratic relationship with the tube voltages; therefore, appropriate tube voltage should be chosen according to the size and weight of the patient in order to reduce the patient dose during the CBCT procedures which obtaining higher image quality. The preset of S20 collimator is preferable for children and petite sized patients, since lower $\mathrm{mAs}$ can be selected to avoid excessive scattered radiation doses. Collimator with smaller longitudinal length settings could also reduce the scanning length, thus the irradiation range and effective dose are considerably reduced simultaneously. Gantry rotation range also affects dose values as well as the dose distribution across the axial plane. The start and stop gantry angles can be deliberately arranged to reduce the sensitive organs involved in irradiation area based upon the disease sites. Longer fields can effectively visualize more anatomy of the patient for the registration process in $3 \mathrm{D}$ CBCT, however, this also results in more doses delivered to a larger volume of the patient with poorer image quality due to the scattering effect of the large scanning coverage area.

For broad beam geometry, the $100 \mathrm{~mm}$ ion chamber will no longer consist of geometrical length to characterize the scattering component from a single-slice profile. They are slightly better surrogate indices for organ dose and thus ultimately for organ risk. The following equation summarized the accumulated dose relationship with the scanning length.

$$
C T D I=\frac{1}{N T} \int_{-50 m m}^{50 m m} D(z) d z<\frac{1}{N T} \int_{-\infty}^{\infty} D(z) d z
$$

Where $\mathrm{D}(\mathrm{z})$ represents the accumulated dose at longitudinal position (z), and NT is the length of integration.

The computed tomography dose index (CTDI) was introduced to optimize the patient dose in CT scan. Although the concept of $\mathrm{CTDI}_{\mathrm{w}}$ was primarily designed for evaluating patient doses from a kilovoltage scanner using fan-beam geometry, the subtle difference in the cone beam setting should not affect the results for cone-beam CT. Therefore, measurements of $\mathrm{CTDI}_{\mathrm{w}}$ remain adequate for CT quality assurance and dose optimization for current LINAC.

The concept of CT radiation dose should be fully understood and appreciated. It is difficult to balance the benefits and possible risks for image guidance in radiation treatment. From one hand, patient will receive additional radiation from the imaging procedure; on the other hand, the accurate positioning due to the image guidance can subsequently reduce the dose to normal tissues. It is undesirable to subtract the imaging dose from the total prescription dose, because the beam quality, dose rate and its radiobiological effect are quite different for each individual disease site. From a clinical point of view, the feasible way is to reduce the imaging dose as low as reasonably achievable. Operators should be adequately informed of all associated risks, and must be familiar with modification techniques according to the patient's clinical situation and not simply just applying preset scanning protocols without patient specific consideration.

\section{Conclusions}

Accurate and precise measurement methods of CTDI for LINAC based integrated CBCT have been 
developed using the CT ion chamber and phantoms. There are many contributing factors that affect the patient imaging doses of a specific CBCT system. Monitoring the patient imaging dose can play a role in achieving excellent imaging at a reasonable radiation dose. It is necessary to change the scanning parameters for different patients to create and combination of best image quality and lowest imaging doses.

\section{References}

[1] D.A. Jaffray, J.H Siewerdsen and J.W. Wong, Flat-panel cone-beam computed tomography for image-guided radiation therapy, International Journal of Radiation Oncology Biology Physics 53 (2002), 1337-1349.

[2] M. Ghilezan, D. Yan and J. Liang, Online image-guided intensity-modulated radiotherapy for prostate cancer: How much improvement can we expect? A theoretical assessment of clinical benefits and potential dose escalation by improving precision and accuracy of radiation delivery, International Journal of Radiation Oncology Biology Physics 60 (2004), 1602-1610.

[3] M.J. Murphy, J. Balter and S. Balter, The management of imaging dose during image-guided radiotherapy: Report of the AAPM Task Group 75, Medical Physics 34 (2007), 4041-63.

[4] M.W. Kan, L.H. Leung, W. Wong and N. Lam, Radiation dose from cone beam computed tomography for imageguided radiation therapy, International Journal of Radiation Oncology Biology Physics 70 (2008), 272-279.

[5] J.R. Perks, J. Lehmann, A.M. Chen, C.C. Yang, R.L. Stern and J.A. Purdy, Comparison of peripheral dose from imageguided radiation therapy (IGRT) using $\mathrm{kV}$ cone beam $\mathrm{CT}$ to intensity-modulated radiation therapy (IMRT), Radiotherapy Oncology 89 (2008), 304-310.

[6] M.K. Islam, T.G. Purdie and B.D. Norringer, Patient dose from kilovoltage cone beam computed tomography imaging in radiation therapy, Medical Physics 33 (2006), 1573-1582.

[7] N. Wen, H. Guan and R. Hammoud, Dose delivered from Varian's CBCT to patients receiving IMRT for prostate cancer, Physics in Medicine and Biology 52 (2007), 2267-2276.

[8] S. Kim, T.T. Yoshizumi, G. Toncheva, S. Yoo and F.F. Yin, Comparison of radiation doses between cone beam CT and multi detector CT: TLD measurements, Radiation Protection Dosimetry 132 (2008), 339-345.

[9] W.Y. Song, S. Kamath, S. Ozawa, S.A. Ani, A. Chvetsov, N. Bhandare, J.R. Palta, C. Liu and J.G. Li, A dose comparison study between XVI and OBI CBCT systems, Medical Physics 35 (2008), 480-486.

[10] A. Amer, T. Marchant, J. Sykes, J. Czajka and C. Moore, Imaging doses from the Elekta Synergy x-ray cone beam CT system, British Journal of Radiology 80 (2007), 476-482.

[11] L.J. Sawyer, S.A. Whittle, E.S. Matthews, H.C. Starritt and T.P. Jupp, Estimation of organ and effective doses resulting from cone beam CT imaging for radiotherapy treatment planning, British Journal of Radiology 82 (2009), $577-584$

[12] L.C. Peng, C.C. Yang, S. Sim, M. Weiss and A. Bielajew, Dose comparison of mgavoltage cone-beam and orthogonalpair portal images, Journal of Applied Clinical Medical Physics 8 (2006), 10-20.

[13] D.E. Hyer, C.F. Serago, S. Kim, J.G. Li and D.E. Hintenlang, An organ and effective dose study of XVI and OBI conebeam CT systems, Journal of Applied Clinical Medical Physics 11 (2010), 181-197.

[14] E.K Osei, B. Schaly and A. Fleck, Dose assessment from an online kilovoltage imaging system in radiation therapy, Journal of Radiological Protection 29 (2009), 37-50.

[15] A. Amer, T. Marchant and J. Sykes, Imaging doses from the Elekta synergy x-ray cone beam CT, British Journal of Radiology 80 (2007), 476-482.

[16] M. Miften, O. Gayou and B. Reitz, IMRT planning and delivery incorporating daily dose from mega-voltage conebeam computed tomography imaging, Medical Physics 34 (2007), 3760-3767.

[17] V. Tsapaki, J.E. Aldrich and R. Sharma, Dose reduction in CT while maintaining diagnostic confidence: Diagnostic reference levels at routine head, chest, and abdominal CT-International Atomic Energy Agency coordinated research project, Radiology 240 (2006), 828-834.

[18] International Commission on Radiation Units and Measurement, Patient dosimetry for X-rays used in medical imaging (Report 74), Journal of the International Commission on Radiation Units and Measurement (2005).

[19] C.B. Saw, Y. Yang and F. Li, Performance characteristic and quality assurance aspects of kilovoltage cone-beam CT on medical linear accelerator, Medical Dosimetry 32 (2007), 80-85.

[20] J. Lehmann, J. Perks and S. Semon, Commissioning experience with cone-beam computed tomography for imageguided radiation therapy, Journal of Applied Clinical Medical Physics 8 (2007), 21-36. 
[21] J. Stutzel, U. Oelfke and S. Nill, A quantitative image quality comparison of four different image guided radiotherapy devices, Radiotherapy Oncology 86 (2008), 20-24.

[22] Y.L. Wang, X.F. Liao and R.G. Ge, Variations of spatial resolution with imaging parameters for kilovoltage conebeam CT, Chinese Journal of Medical Imaging Technology 26 (2010), 1173-75. 